\section{The role of accommodation in the control of binocular rivalry}

\author{
LEON C. LACK \\ Flinders University of South Australia, Bedford Park, South Australia 5042
}

The role of accommodation in the control of binocular rivalry (BR) has been granted various degrees of importance by $\mathrm{Es}$ in the past. The most recent investigation by Fry (1936) concluded that accommodation provides the basis of BR control through the blurring of retinal images. However, the present study found that the introduction of very small artificial pupils $(0.5 \mathrm{~mm})$ did not reduce $B R$ control. It was concluded that if accommodation changes are occurring with large pupils, the resulting image blurring plays no part in control of rivalry. Experiment 2 tested the effect of paralyzed intrinsic eye muscles and found almost the same degree of control as in the normal state. The slight decrease of control that was present was attributed to a general performance decrement, since slight performance decrements with eye paralysis were also found in a visual reaction-time task and hand dynamometer test. In Experiment 3, it was found that the increased control that was obtained over several practice sessions was mostly retained during subsequent eye paralysis. These findings and, in addition, a very significant control of rivaling afterimage stimuli under eye paralysis strongly suggest a central component of BR control rather than one based on accommodation.

If dissimilar patterns stimulate corresponding retinal areas of the two eyes, the observer perceives an alternation between these two patterns known as binocular rivalry (BR). Helmholtz (1925), Breese (1899), McDougall (1903), DeVries and Washburn (1909), and Washburn and Gilette (1933) have shown that the predominance of one of the patterns could be increased temporarily by a voluntary effort as compared to the passive viewing condition. George (1936), Fry (1936), Meredith and Mexedith (1962), and Lack (1969) have shown that control can also be demonstrated by decreasing or increasing the rate of alternation as compared with the rate during passive viewing. Helmholtz (1925) classified $\mathrm{BR}$ as a phenomenon of alternating attention, and attributed $\mathrm{BR}$ control to the willful concentration of attention. Despite the potential significance of BR control to the study of attention, there have been only a few experiments, of dubious value, and no recent experiments to investigate the mechanism of $B R$ control.

Most concern in the past has been with the role of ocular accommodation as the mediator of $B R$ control. McDougall (1903) tested the effect of accommodation in BR by paralyzing the intrinsic eye muscles of one eye with the use of atropine. The subsequent predominance of the right eye was attributed to the loss of accommodation ability in the left eye. By voluntary control, McDougall (1903) could increase the predominance of either the right eye or paralyzed left eye. The extent of increased predominance for the right eye was slightly greater than that for the left eye but was proportionately similar to the original passive predominance of each eye. McDougall (1906) concluded that even in the normal conditions, differential accommodation activity between the two eyes would play at the most a secondary role in the control of attention in BR.

George (1936), in a series of tests measuring reversible perspective and $B R$ rates, mentioned briefly the effects of paralyzing the intrinsic eye muscles. This had "relatively little effect on voluntary control in tests of reversible perspective, but in tests of binocular rivalry the control was noticeably decreased." Intrinsic eye muscle paralysis also "noticeably affected" the voluntary control of rivaling afterimages. Rather than attribute this decrease of $B R$ control specifically to the loss of accommodation, he made the more general conclusion that $\mathrm{BR}$ is a "lower level function" than that of perspective reversal. Unfortunately, since George (1936) provides no data or statistics, it is impossible to assess the degree to which BR control was affected by eye paralysis in his experiment.

Fry (1936), in a rather bold interpretation of the earlier articles, assumed that accommodation "provides the basis for the voluntary suppression of vision in one eye or in the favoring of vision in the other." Hence, accommodation would be the basis of BR control. He found that BR control was nearly abolished in his $S$ when small artificial pupils were used. improbable case of its existence in
Therefore, he concluded that in the case of direct stimulation of the two eyes with rivalry patterns, BR control is effected through accommodation by the blurring of retinal images.

However, Fry (1936) has not in fact shown that accommodation changes do take place during $B R$ control with the eyes in the normal unparalyzed state. He infers that accommodation is the basis of BR control because control is lost when his S's eyes are paralyzed. However, neither McDougall (1903) nor George (1936) found BR control to be obliterated by paralyzing the intrinsic eye muscles. Indeed, McDougall found hardly any effect and warned (1906) against the overemphasis of peripheral motor systems in the control of attention.

Both Fry (1936) and McDougall (1903) reported the data from one experimental S-presumably themselves in each case. In studying factors affecting voluntary control of attention, it is obviously desirable to minimize 'Ss' expectations and to take care to control motivation or instructional conditions. A necessary prerequisite would be to use a sufficient number of Ss naive to the purposes of the experiment. The purpose of the following experiments was to overcome past experimental deficiencies in clarifying the role of accommodation in BR control.

\section{EXPERIMENT 1}

The first experiment was to test the effect of artificial pupils on BR control. Fry (1936) found that the reduction of artificial pupil size from 3.94 - to $2.06-\mathrm{mm}$ pupils was sufficient to abolish control in his $\mathrm{S}$. In any case, with artificial pupils smaller than $0.5 \mathrm{~mm}$ in diam, BR control would necessarily be abolished, since accommodation changes would become completely ineffective in blurring the retinal images. In the present experiment, Ss were tested for their degree of control of BR under the conditions of no artificial pupils, and with $2.8-\mathrm{mm}$ artificial pupils, 1.7-, $1.0-$, and $0.5-\mathrm{mm}$ pupils.

\section{Method}

Subjects. Nine (five males and four females) volunteer Ss were obtained from the nonacademic staff of the Psychology Department. All Ss had normal vision without the use of corrective lenses. The natural pupil diameters of all Ss were measured to the nearest $0.5 \mathrm{~mm}$ in the viewing condition of no artificial pupils. The mean natural pupil size was $5.7 \mathrm{~mm}$, with no $S$ having natural pupils less than $4.5 \mathrm{~mm}$ in diam. Thus, it seems reasonable to assume that all the artificial pupil conditions produced reductions of effective pupil size in all 
Table 1

Mean Slow Rates and Rapid Rates in Alternations Per Minute and Mean BR Control Percentages for the Six Conditions of Both Test Sessions

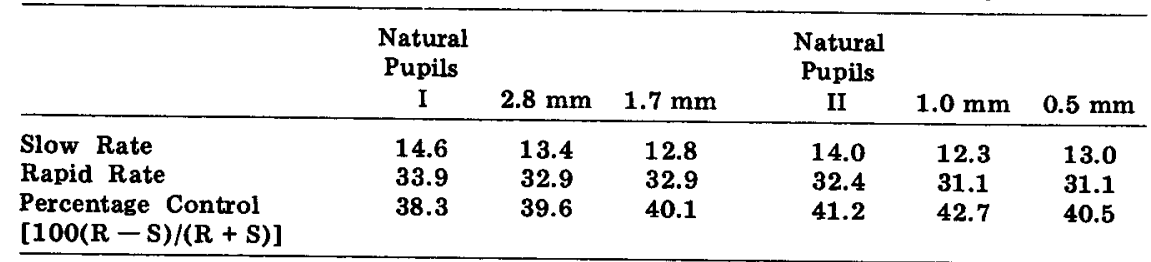

Ss. No Ss had ever practiced BR control, and only three had any previous experience of BR. All Ss were naive to the purpose of the experiment.

Stimuli. The fusion stimuli consisted of left and right black rings, $58 \mathrm{~mm}$ apart, with inner diameters of $8.5 \mathrm{deg}$ and outer diameters of 10.5 deg. Each ring was centered on a 28-deg square white field. The white fields were on black surrounds of a stereocard which provided a second fusion contour for the two fields. The rivalry contours were a black vertical diameter, 1 deg in width, in the right fusion ring and a black horizontal diameter, 1 deg in width, in the left fusion ring. The fusion rings and rivalry lines were inked on thin white paper and mounted in the black stereocard.

Apparatus and procedure. The apparatus consisted mainly of a Stereoking Model HN-44 stereoscope, in which could be fixed a $4 \times 10 \mathrm{~cm}$ stereocard, $5.5 \mathrm{~cm}$ from refracting lenses, providing fixation at infinity. Immediately in front of the refracting lenses, artificial pupils could be mounted and adjusted to the intraocular distance of any $S$. The stimuli were transilluminated by a $500-\mathrm{W}$ incandescent bulb placed behind the stereocard. To keep retinal illumination constant for all pupil conditions, adjustments were made in the distance of the incandescent bulb from the stereocard and the input voltage to the bulb by the use of a Variac transformer. The effective luminance was measured at the lens for the no-pupil condition or behind the artificial pupil with an S.E.I. spot photometer. In all conditions, the luminance of the black fusion rings, vertical, and horizontal diameters was $0.31 \mathrm{fL}$. The white background luminance was a uniform $4.0 \mathrm{fL}$. A chinrest and nose slot were adjusted for the $S$ in order to provide a comfortable and fairly stable head position. During a test trial, $\mathbf{S}$ pressed a small event counter in his preferred hand to indicate each alternation in BR. Rate of alternation was measured for $30-\mathrm{sec}$ timed trials which were separated by 30 -sec rest periods.

All Ss were given the general instructions: (1) "at the (ready) signal look into the stereoscope and fixate at the apparent intersection point of the vertical and horizontal lines," (2) "after the (start) signal press the counter once for each alternation in rivalry," and (3) "at the (stop) signal stop pressing and look away." Ss were instructed to keep their heads stationary and not to blink excessively during test trials. In addition, Ss received one of three different instructions for any one test trial. The instructions were identical to those detailed in an earlier article by the present author (Lack, 1969) of "passive rate," "slow rate," and "rapid rate." The "slow rate" and "rapid rate" instructions were essentially the same as Fry's (1936) "attempted retardation", and "attempted acceleration" conditions. They were then given 2-min viewing in the no-pupil condition to familiarize them with counting alternations during $B R$ and to allow the $B R$ rate to stabilize after what Cogan and Goldstein (1967) found to be an initial increase of BR rate commonly observed in naive Ss. In each viewing condition, all Ss followed the same sequence of instructions over nine test trials: the "passive rate" for the first 30-sec test trial, the "slow rate" for the next four trials, and the "rapid rate" for the last four trials. Initially, all nine Ss were tested under the three viewing conditions of no artificial pupils, 2.8-mm pupils, and $1.7-\mathrm{mm}$ pupils in balanced order so that each condition appeared three times in each position of order. Approximately 2 months later, all nine Ss were tested in the viewing conditions of no artificial pupils, $1.0-\dot{\mathrm{mm}}$ pupils, and $0.5-\mathrm{mm}$ pupils in a similar balanced order. A complete testing session was about $45 \mathrm{~min}$ in duration. Ss were given no knowledge of results throughout the experiment.

\section{Results and Discussion}

After the initial 2-min familiarization period, all Ss reported no difficulty in indicating rivalry alternations of the vertical and horizontal lines. E observed no head movements of Ss during all the test trials. Ss also followed the instructions not to blink excessively. Most Ss, in fact, did not blink at all during any 30-sec test trial. Ss who did blink occasionally during test trials did so at about the same low frequency regardless of the viewing condition or instructions.

The measure of rivalry rate under the "slow rate" and "rapid rate" instructions in alternations per minute and the percentage $B R$ control measure, $(100)$ (rapid - slow)/(rapid + slow), were the same as described in a previous article (1969).

Table 1 gives the mean slow rates, rapid rates, and percentage control measures for both test sessions. Since there was no practice effect between the two test sessions, presentation order was not included in the subsequent data analysis. An analysis of variance applied to the slow rate and rapid rate measures showed a significant difference ( $F=27.9$ $\mathrm{df}=1 / 8, \mathrm{p}<.001$ ) between the rivalry rates but no significant effect $(\mathrm{F}=0.98, \quad \mathrm{df}=5 / 40, \quad \mathrm{p}>.50)$ of pupillary conditions on the rivalry rate measures. A S by Condition analysis of variance applied separately to the BR control measures found no significant effect $(F=0.11$, df $=5 / 40, p>.50)$ due to pupillary conditions. In summary, the instructions of "slow rate" and "rapid rate" consistently produced a very significant difference of rivalry rates in all pupillary conditions. In this experiment, the reduction of artificial pupil size had no effect on BR control.

The discrepancy of the present results with Fry's (1936) findings are unlikely to be due to differences in stimulus conditions. The retinal area subtended and the black-white contrasts were about the same in both cases. The main difference in stimulus conditions was that Fry (1936) had three bars in each rivalry stimulus compared to one bar in the present experiment. In any case, according to the retinal blurring hypothesis, the $\mathrm{BR}$ control of any illuminated stimuli should be lost with the use of the very small artificial pupils.

A second source of discrepancy may be due to differences in the experimental Ss. As far as possible, the Ss in the present experiment were kept naive and free from expectations about their ability to control the rivalry in any of the pupillary viewing conditions. The fact that the overall mean slow rate, rapid rate, and percentage control is nearly identical to these respective measures in a naive unpracticed group of eight $\mathrm{Ss}$ in the similar conditions of an earlier experiment (Lack, 1969) suggests that the Ss in the present experiment were a representative naive sample. On the other hand, any prior expectations on the part of Fry's (1936) $S$ may have made it difficult for him to exert equal 
amounts of effort under the two pupillary conditions. Whatever the case, the reliability of Fry's (1936) results with respect to the effect of small artificial pupils on BR control would now seem to be in question.

The lack of any difference due to artificial pupils does not exclude the possibility that accommodation changes do provide the basis of $B R$ control. It only suggests that if there are accommodation changes concomitant with $B R$ control, the resulting image blurring in the natural pupil or large pupil condition has no effect on BR control as compared to the small artificial pupil condition in which retinal image blurring is eliminated. One is left with the necessity of developing alternative explanations if accommodation is still held as the basis of BR control. In any case, BR control should be abolished when the accommodation function is eliminated due to paralysis of the intrinsic eye muscles. The next experiment, therefore, examines the effect of intrinsic eye muscle paralysis on BR control.

\section{EXPERIMENT 2}

A mydriatic and cycloplegic named Mydrilate was used. One drop in each eye was sufficient to produce complete ciliary muscle paralysis and pupillary dilation commencing approximately $15 \mathrm{~min}$ after administration and lasting approximately $6 \mathrm{~h}$. Its effects could be more rapidly reversed after a testing session with the administration of one drop in each eye of $0.5 \%$ solution physostigmine. The present author initially tested the effect of Mydrilate on himself and observed that the inability to focus was subjectively disturbing and seemed to produce increased caution. These introspections suggest that intrinsic eye muscle paralysis may produce a generalized motivational decrement in Ss. To test for this possibility, a visual task (simple reaction time, RT) and a nonvisual task (hand squeeze strength) were included in addition to the test of BR control. If BR control was abolished but the RT task and squeeze strength task were unchanged with Mydrilate, it would be clear confirmation of accommodation as the basis of BR control.

\section{Method}

Subjects. Twenty (10 males and 10 females) $S$ s with uncorrected normal vision and under 30 years of age were obtained from an introductory psychology class. No $S$ had an:' prior experience with BR. All were naive as to the purpose of the experiment.

Stimuli. The apparatus, illumination, and testing procedure for

Table 2

Means for Both Groups Combined of Rivalry Rates, Percentage BR Control, Reaction Time, and Squeeze Strength Under Normal Conditions and With the Intrinsic Eye Muscles Paralyzed

\begin{tabular}{llcccc}
\hline & $\begin{array}{c}\text { Slow } \\
\text { (Alternations/Min) }\end{array}$ & $\begin{array}{c}\text { BR } \\
\text { Control } \\
\text { (Percent) }\end{array}$ & $\begin{array}{c}\text { RT } \\
\text { (Msec) }\end{array}$ & $\begin{array}{c}\text { Squeeze } \\
\text { Strength } \\
\text { (lbs/in. }^{2} \text { ) }\end{array}$ \\
\hline Normal & 12.6 & 32.8 & 42.8 & 260 & 18.4 \\
Paralyzed & 13.8 & 30.5 & 37.9 & 269 & 17.8 \\
Difference & n.s. & $<.10$ & $<.10$ & $<.05$ & $<.10$ \\
\hline
\end{tabular}

BR control were the same as that in Experiment 1, except that only the $0.5-\dot{m m}$ artificial pupils were used. In addition, Ss were instructed to maintain for all three instructional conditions the same criterion of what they considered to be a BR alternation. This instruction was to test the extent to which $S s$ in Experiment 1 may have enhanced their BR control measures by distorting their criterion under the "slow rate" and "rapid rate" instructions.

The apparatus for the visual RT task consisted of telegraph key, reaction stimulus, reaction timer, and electrical circuitry, in addition to the chinrest and stereoscope used in the BR task. Ss viewed the stimulus through the stereoscope with the stereocard and artificial pupils removed. The stimulus (a 24-V $3-\mathrm{W}$ red light bulb placed $60 \mathrm{~cm}$ behind the stereoscope) provided a binocularly viewed red disk of 25-fL uniform illumination. Ss were positioned at the stereoscope in a lightproof and sound-deadened room. $S$ was instructed to hold the fingers of his preferred hand immediately above the telegraph key and to respond as quickly as possible at the onset of the reaction stimulus by tapping the telegraph key. Thirty-five reaction trials were presented in serial order with no warning signals. The onset of reaction stimuli followed previous trials by varying intervals of time on a semirandom schedule. The intertrial intervals of $3,4,5,6$, and $7 \mathrm{sec}$ occurred an equal number of times in the total of 35 trials. Ss were warned to wait for the onset of the reaction stimulus to avoid anticipatory tapping.

The apparatus for the squeeze task consisted simply of a hand dynamometer hydraulically connected to a pressure gauge. Ss were instructed to squeeze the dynamometer bulb as strenuously as possible on each of three trials, separated by 30 -sec rest intervals.

All Ss performed the three tasks in the same order (BR control, RT task, and squeeze strength task) on each of two separate test sessions held 1 week apart at the same time of day. For both sessions, the instructions and procedures were exactly the same except that in one session Ss had
Mydrilate administered $20 \mathrm{~min}$ prior to testing.

The $20 \mathrm{Ss}$ were divided randomly into two groups so that both Group $A$ and Group B contained five males and five females. Group A first had the normal test session followed by the treatment test session; Group B had the reverse order of conditions. At no time were any Ss given any knowledge of results.

\section{Results and Discussion}

In the BR task the same measures as used in Experiment 1 were taken of passive rate, slow rate, and rapid rate in alternations per minute, and the percentage $B R$ control calculated from the formula $(100)(R-S) /(R+S)$. In the RT task, the first 10 trials from each $S$ in each test session were considered as warm-up trials and were excluded from the results. The median RT from the remaining 25 trials was used as the S's RT in each test session. In the squeeze strength task, the mean of the three trials in lbs/in. ${ }^{2}$ was used as the S's squeeze strength for each test session.

The mean values of each of these measures for the 20 Ss under each treatment condition are shown in Table 2.

The effect of intrinsic eye muscle paralysis tends to be the same as that found by Fry (1936)-a reduction of rapid rate and an increase of slow rate. However, the changes are so slight that neither reaches the .05 level of significance with a one-tailed test. The mean reduction of the $B R$ control measure approached significance $(t=1.62$, df $=19, .10>p>.05)$, but the mean increase of reaction time is significant $(t=1.87 ; \mathrm{df}=19, \mathrm{p}<.05)$, and the mean decrease of squeeze strength also approaches significance $(\mathrm{t}=1.64, \quad \mathrm{df}=19, \quad .10>\mathrm{p}>.05)$. Therefore, the effect of eye paralysis produces a slight decrement in performance, which reaches about the same level of significance for all three tasks. The differences between the mean slow rate and mean rapid rate is still very significant $(t=6.5, d f=19$, $\mathrm{p}<.0001)$ in the eyes-paralyzed condition.

The only difference in procedure between the otherwise identical testing conditions of the Experiment 1 
0.5-mm pupil condition and Group A in the normal condition of the present experiment was the additional instruction given to Group $A$ to use the same criterion for alternations under both rivalry rate instructions. The two groups have almost identical mean values of slow rate, rapid rate, and $\mathrm{BR}$ control. Therefore, it seems reasonable to conclude that $\mathrm{Ss}$ do not distort their criterion to obtain fewer alternations in the slow rate and more alternations in the rapid rate.

In conclusion, intrinsic eye muscle paralysis does not produce a specific loss of BR control. On the contrary, very significant $B R$ control is present with the complete abolition of accommodation activity. If the nonsignificant tendency of decreased BR control with eye paralysis is real, it is probably due to some central factor that produced performance decrements in all three tasks.

\section{EXPERIMENT 3}

Fry (1936) found that BR control of afterimage stimuli was practically abolished with the use of homatropine. He reasoned that $\mathrm{BR}$ control of afterimages was mediated through the intraocular pressure changes resulting from accommodation. Although research investigating the effects of accommodation on intraocular pressure (Armaly \& Rubin, 1961) did not find the sort of pressure-changing mechanism envisaged by Fry, it is still possible that intrinsic eye muscle paralysis does abolish control of afterimage rivalry. Therefore, this possibility needed to be reinvestigated. It was also decided to include a test of the comparative controllability of single line and triple line rivalry stimuli during eye paralysis to determine if the discrepancy of the findings of the present experiments with Fry's (1936) was due to the difference in rivalry stimuli,

The present author (1969) found that spaced practice of the "slow rate" and "rapid rate" instructions produced a decrease in the measured slow rate, an increase in the measured rapid rate, and hence an increase in the measure of $\mathrm{BR}$ control. Because this result was obtained with eyes normal and without the use of small artificial pupils, it is possible that an accommodation mechanism provided the basis for the increased control of well-practiced Ss. This possibility was also tested in the present experiment.

\section{Method}

Subjects. Twenty-eight (14 males and 14 females) Ss with uncorrected normal vision and $r$ nder 30 years of age were obtaned from an introductory psychology class. All Ss were naive with respect to $B R$ and the purposes of the experiment.

Stimuli. One pair of rivalry stimuli (single vertical and horizontal diameters of fusion rings, $\mathrm{H}-\mathrm{V}$ ), was the same as that of Experiments 1 and 2. A second pair of rivalry stimuli (3H-3V) was produced by adding two equally spaced lines parallel to each of the rivalry diameters of the first pair. The additional rivalry lines were $7 \mathrm{deg}$ in length and 1 deg in width and had the same illumination as the other black fusion and rivalry lines. Instead of producing one intersection point of rivalry as the first rivalry pair, this pair produced the intersection of three vertical and three horizontal lines, or nine rivalry intersections. The afterimage rivalry stimuli consisted of a single vertical bar on the right eye and a single horizontal bar on the left eye, which bisected each other in the binocular condition. Both bars were $4 \mathrm{deg}$ in width and $28 \mathrm{deg}$ in length. They were produced by a photoflash behind the stereoscope projected through cut-out slots of a black stereocard.

Apparatus and procedure. The apparatus, instructions, and stimulus illumination for the two pairs of illuminated rivalry stimuli were the same as in Experiment 2. The apparatus for the test of afterimage rivalry control was the same as that of Experiments 1 and 2, except that the artificial pupils were removed and the black stereocard with the vertical and horizontal bar slots was inserted in the stereoscope. A Blaupunkt manually triggered photoflash was mounted $20 \mathrm{~cm}$ behind the stereoscope equidistant from the two eyepieces. It produced a flash with a constant output of $150 \mathrm{~J}$ for a duration of approximately $1 \mathrm{msec}$. The Ss were instructed to close their eyes immediately after the flash and to view the afterimages. The "start" signal for the beginning of rivalry alternation counting was given always $5 \mathrm{sec}$ after the flash.

The 28 Ss were allotted randomly to two groups so that a well-practiced Group $P$ and an unpracticed Group $U$ each contained seven males and seven females. Group $P$ was initially tested with Mydrilate, using only the single vertical and horizontal rivalry stimuli, and without knowledge of results. Group $P$ was then given 12 consecutive practice days with eyes normal. Prior to each practice day after Practice Day 1, each $\mathbf{S}$ was given knowledge of his previous day's performance in terms of mean slow rate, mean rapid rate, and percentage $B R$ control. $S$ was then given knowledge of results after each trial and was reinforced with mild praise for any improved performance at the end of the practice day.
According to the findings of the author (Lack, 1970), this procedure gives the maximum increase of $B R$ control. Following the 12th practice day, Group $P$ was given a final test day with Mydrilate and without knowledge of results. Ss were tested with the single line rivalry stimuli, then triple line rivalry stimuli, and finally with the afterimage rivalry stimuli, following the normal test trial sequence in each case. For the afterimage stimuli, the $30-\mathrm{sec}$ rest periods were extended to $100 \mathrm{sec}$. Group $U$ was tested under the same conditions and procedure as the final test day of Group P.

\section{Results and Discussion}

The Ss found no difficulty in indicating $\mathrm{BR}$ alternations of the afterimage stimuli on the final test day. The alternations were reported as being unambiguous, with a complete disappearance of one afterimage bar usually occurring with the appearance of the other. This virtually eliminated the possibility of criterion problems for Ss.

The main results of this experiment are illustrated in Fig. 1: Group P's practice of the "slow rate" and "rapid rate" instructions showed very significant effects between Practice Day 1 and Practice Day 12. The mean slow rate showed a very significant decrease $(t=9.73, \quad d f=13$, p <.0001); the mean rapid rate showed a very significant increase $(t=11.2, d f=13, p<.0001) ;$ and the percentage control measure showed a very significant increase $(t=21.0$, df $=13, p<.0001)$. The effects due to practice are very similar to the results of the author's earlier studies (Lack, $1969,1970)$. The fact that this increase of BR control, using the $0.5-\mathrm{mm}$ pupils, is at least as great as that without artificial pupils is further confirmation of the conclusions of Experiment 1.

Comparisons between the initial test day and Practice Day 1 found no significant differences of slow rate, rapid rate, and $B R$ control. This confirms the results of Experiment 2, that eye muscle paralysis has no effect on unpracticed control. On the other hand, the differences between Practice Day 12 and the final test day did reach significance $(p<.01)$. It is doubtful if these differences can be attributed to a specific loss of an accommodation role in well-practiced control because there are other factors which would tend to decrease the performance of Group $P$ on the final test day. A general performance decrement with Mydrilate would be expected to produce the same proportional decrement with unpracticed control, but this would produce a greater 


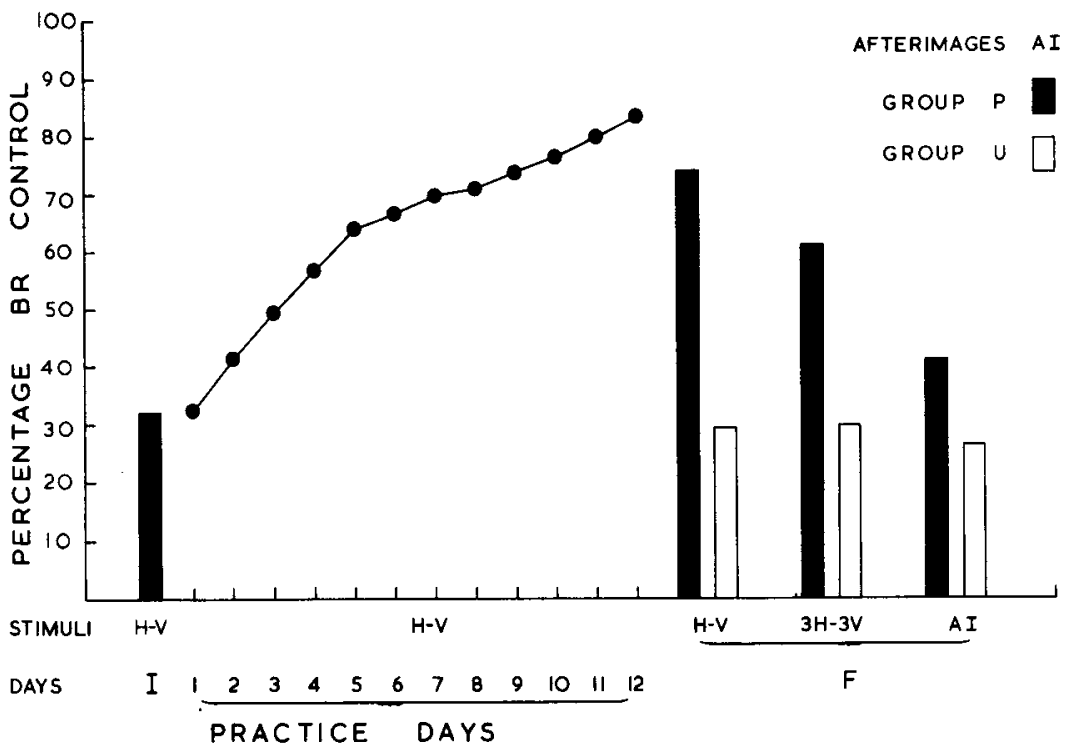

Fig. 1. Mean BR control percentages with eyes paralyzed for Group $P$ on the initial test day (I) and for Group P and Group $U$ on the final test day (F) and means for Group $P$ with eyes normal on the 12 practice days.

absolute reduction of well-practiced control. The absence of knowledge of results and reinforcement on the final test day must also tend to decrease Group P's performance in comparison with Practice Day 12.

Although the effect of eye paralysis is a significant decrease of $\mathrm{BR}$ control from a mean of $84.0 \%$ on Day 12 to a mean of $74.3 \%$ on the final test day, a comparison with the initial test day mean of $32.0 \%$ shows that what control was developed through practice has been mostly retained. The comparison between the initial test day and final test day for Group P showed very significant $(p<.0005)$ differences of slow rate, rapid rate, and BR control, as did the comparison between the unpracticed Group $U$ and the Group P final test day. In summary, the practice of "slow rate" and "rapid rate" instructions with eyes normal produces a very significant increase of control between the initial and final eyes-paralyzed conditions. In addition, it seems unlikely that this increased control is dependent on accommodation.

The effect of practice with the H-V stimuli clearly transferred to the control of the $3 \mathrm{H}-3 \mathrm{~V}$ stimuli and afterimage stimuli since the $B R$ control measures were significantly
In any case, he is implying that the main source of control still accessible to $\mathrm{BR}$ is a component that can be affected on a higher level. This corresponds with MeDougall's (1906) conclusions that despite the slight effect of atropine in reducing voluntary attention in $B R$, the role of motor adjustment of sense organs is one that is only secondary to what he calls "cerebro-ideational activity." The results of the present experiments also suggest that the peripheral mechanisms of accommodation, pupillary activity, blinking, and retinal image movement, if they have a real effect at all, play only a secondary role in the control of binocular rivalry.

\section{REFERENCES}

ARMALY, M. F., \& RUBIN, M. E. Accommodation and applanation tonometry. Archives of Ophthalmology, $1961,65,415-423$.

BREESE, B. B. On inhibition. Psychological Monographs, 1899, 3, 1-65.

COGAN, R \& GOLDSTEIN, A. G. The stability of binocular rivalry during spaced and massed viewing. Perception \& Psychophysics, 1967, 2, 171-174.

De VRIES, A. \& WASHBURN, M. F. Minor studies from the Psychological Laboratory of Vassar College: X. A study of retinal rivalry in the after-image. American Journal of Psychology, 1909, 20,131-138.

FRY, G. A. The relation of accommodation to the suppression of vision in one eye. American Journal of Ophthalmology, 1936, 19, 135-138.

GEORGE, R. W. The significance of the fluctuation experienced in observing ambiguous figures and in binocular rivalry. Journal of General Psychology, $1936,15,39-61$.

HELMHOLTZ, $\mathrm{H}$. von. Treatise on physiological optics, Vol, 3. J. P. Southall (Ed.) New York: Dover, 1925.

LACK, L. C. The effect of practice on binocular rivalry control. Perception \& Psychoph ysics, 1969, 6, 397-400.

LACK, L. C. Effect of knowledge of results and spacing of practice trials in training of control of binocular rivalry. Perceptual \& Motor Skills. 1970, 31, 827-830.

MCDOUGALL, W. Physiological factors of the attention process (III). Mind, 1903, 12, 473-488.

MCDOUGALL, W. Physiological factors of the attention process (IV). Mind, 1906, $15,329-359$.

MEREDITH, G. M., \& MEREDITH, C. G. W. Effect of instructional conditions on rate of binocular rivalry. Perceptual \& Motor Skills, 1962, 15, 655-664.

WASHBURN, $M_{\circ}$ F., \& GILETTE, A. Studies from "the Psychological Laboratory of Vassar College: LXII. Motor factors in voluntary control of cube perspective fluctuations and retinal rivalry fluctuations. American Journal of Psychology, 1933, 45, 315-319.

(Accepted for publication January 10, 1971.) 